\title{
Arterial blood pressure response to head-up tilt test and orthostatic tolerance in nurses
}

\author{
Chisato Onizuka $^{1} \cdot$ Yuki Niimi $^{2} \cdot$ Motohiko Sato $^{1} \cdot$ Junichi Sugenoya $^{1,3}$
}

Received: 6 October 2014/Accepted: 2 March 2015/Published online: 17 April 2015

(C) The Japanese Society for Hygiene 2015

\begin{abstract}
Objectives High tolerance to postural changes was examined in nurses.

Methods Twelve female nurses and 12 healthy controls underwent a $70^{\circ}$ head-up tilt (HUT) test for $10 \mathrm{~min}$. Blood pressure (BP), heart rate (HR), pulse pressure, and hormone levels were measured. Baroreceptor sensitivity (BRS) was calculated using a sequence technique.

Results HR increased during HUT in both subject groups, with no difference between groups. Systolic BP was rapidly increased by HUT in both subject groups, and was higher in the nurse group than in the control group during the first 2 min of HUT. Pulse pressure decreased during 1-2.5 min of HUT in the control group, but there was no decrease in the nurse group. BRS was decreased by HUT in the nurse group, while it tended to be decreased in the control group. Both during baseline and HUT, BRS was lower in the nurse group than in the control group. Plasma noradrenaline increased with HUT, and the increase was greater in the nurse group than in the control group.

Conclusions Although nurse subjects had a lower BRS during HUT than control subjects, they were able to effectively maintain BP during HUT, suggesting that nurse subjects had higher orthostatic tolerance. The better maintenance of $\mathrm{BP}$ in nurse subjects appeared to be
\end{abstract}

Chisato Onizuka

onizuka@aichi-med-u.ac.jp

1 Department of Physiology, Aichi Medical University, Nagakute, Aichi 480-1195, Japan

2 Division of Neurology, Japanese Red Cross Nagoya First Hospital, Aichi 453-8511, Japan

3 School of Nursing, Sugiyama Jogakuen University, Aichi 464-8662, Japan associated with a compensatory mechanism other than the arterial baroreflex and/or a hemodynamic mechanism.

Keywords Baroreflex - Blood pressure - Head-up tilt . Nurse · Orthostatic tolerance

\section{Introduction}

Typical work for nurses tends to have a high degree of physical activity. For example, a study reported that energy expenditure for physical activity performed on the working days of clinical nurses was 1.5 times more than that of most office workers [1]. Studies using motion analysis found that the routine work of nurses is characterized by assuming a standing position for long periods of time and by frequently changing the body posture throughout the working time [2, 3]. This indicates that it might be advantageous for nurses to have a high tolerance to orthostasis because, if tolerance is low, the ability to perform their duties could be diminished. However, to our knowledge, orthostatic tolerance of nurses has not been studied.

When body posture is changed from a supine to upright position, blood shifts to the lower part of the body. This blood shift then reduces the venous return, so that the cardiac output and blood pressure (BP) tend to decline. This BP reduction, however, is compensated partly by various reflex mechanisms including arterial baroreflex $[4$, 5], cardiopulmonary reflex [6, 7], vestibulosympathetic reflex [8], or venoarteriolar axon reflex [9], and thus BP is maintained during an upright posture. Improvement in orthostatic tolerance is achieved by improving orthostatic cardiovascular responses [10]. For example, exercise training (moderate, but not severe) can improve orthostatic tolerance [11]; the underlying mechanism is assumed to 
involve increased blood volume $[11,12]$, reduced leg venous compliance [13], and an altered baroreflex function $[11,13]$.

Among compensatory mechanisms, the arterial baroreflex plays a primary role in the maintenance of BP during posture change [5]. Arterial baroreflex function is usually evaluated by baroreflex sensitivity (BRS), which is defined as the slope of the baroreflex function curve. The baroreflex function curve describes an input-output relationship of the baroreflex, and is represented as a (inverse) sigmoidal curve [14]. In addition, arterial baroreflex function includes the mechanism of "resetting" $[15,16]$, by which the baroreflex function curve can shift horizontally or vertically [15-17]. In fact, it has been demonstrated that HUT may induce a rightward shift of the function curve to increase sympathetic vasoconstriction, and thereby protect against hypotension [17, 18].

In this study, we hypothesized that nurses have an improved orthostatic tolerance because of excellent cardiovascular responses to orthostasis. Therefore, we examined the responses of BP, heart rate (HR), several hormones related to cardiovascular regulation, and arterial baroreflex sensitivity (BRS) to the HUT test, and the results were compared between the nurse and control subjects.

\section{Methods}

\section{Subjects}

Participants in this study were recruited according to the Declaration of Helsinki. All participants thoroughly understood the contents provided by oral and written explanation, and gave their written consent. The experiments were conducted with sufficient safety considerations. This study was conducted under the approval of the Human Subjects Ethics Committee of the School of Medicine, Aichi Medical University, and the Ethical Committee of the Japanese Red Cross Nagoya Daiichi Hospital.

Subjects were recruited by posting a public notice in several universities and a hospital. A total of 24 female subjects, which included 12 healthy nurses and 12 healthy controls, volunteered. They had no underlying diseases and no history of syncope, and had regular menstrual cycles. They were not athletes and had no habits of daily exercise. They did not smoke. All 12 subjects in the nurse group had worked in the hospital emergency ward on three different shifts (day, evening, and night) for more than 3 consecutive years, and they had worked as a nurse for 3-14 years. Control subjects were all graduate students, and their daily life involved research activity in life sciences at the university and occasional light work in part-time jobs. The activity level of the control subjects in their private out-of- work hours was estimated to be fundamentally comparable with that of the nurse subjects. However, the activity level of the control subjects during work hours was estimated to be equivalent to an ordinary office worker. None of the control subjects had ever experienced night shift work.

\section{Experimental protocol}

The HUT test was performed using a motorized tilt table according to the guidelines provided by the American College of Cardiology Expert Consensus [19]. HUT testing was performed in a sound proof room at an air temperature of $26.4 \pm 0.1$ [SE] ${ }^{\circ} \mathrm{C}$, and humidity of $39 \pm 3 \%$. Measurements were made within 7 days after the initiation of menstruation. Before starting the experiments, each subject was informed of the measurement method using the tilt table, and subsequently underwent body tilting on the table to familiarize themselves with the procedure.

Measurements were performed at 18:00, during the offshift period that intervened between a day shift and the subsequent night shift. Subjects were required to abstain from eating for $3 \mathrm{~h}$ and from drinking about 30 min prior to the test. They were also asked to urinate $1 \mathrm{~h}$ prior to the test.

The test subject, after having entered the test room, rested supine on the tilt table. Electrocardiograph (ECG) electrodes and a BP cuff were attached, and ECG and BP were recorded continuously. An intravenous route was then constructed for blood sampling through the left cephalic vein using a heparin sodium-locked syringe (Otsuka Pharmaceutical Co, Tokyo, Japan). After the supine rest for $30 \mathrm{~min}$, a blood sample of about $20 \mathrm{ml}$ was taken. When $\mathrm{HR}$ and BP became stable, the tilt table was elevated to a $70^{\circ}$ angle in $30 \mathrm{~s}$. At $10 \mathrm{~min}$ of HUT, another blood sample was taken, and the tilt table was returned to the $0^{\circ}$ position. Measurements were continued further for $10 \mathrm{~min}$.

The test was terminated according to the criterion of the development of presyncopal signs and symptoms such as a drop in arterial BP below $80 \mathrm{mmHg}$, discomfort, dizziness, nausea, sweating, or the subject request to discontinue the test. Subjects who discontinued the test were asked whether they agreed to repeat it on a later day.

\section{Measurement of heart rate and BP}

ECG data were recorded using a bioamplifier (AB621G, Nihon Kohden, Tokyo, Japan). Signals were sampled at $1000 \mathrm{~Hz}$ via an A/D converter. Thus, digitalized ECG signals were stored in a computer and later processed using Tonam2C software (Tonam2C Version 1.0 for Windows, Suwa Trust Ltd, Tokyo, Japan) to obtain BRS.

BP (systolic blood pressure SBP; and diastolic blood pressure, DBP) was measured beat-to-beat using digital 
photoplethysmography (Finapres [20, 21], Model 2300, Ohmeda, Tokyo, Japan). The cuff was placed on the second phalanx of the third finger of the subject's dominant hand, which was positioned at the height corresponding to the heart throughout the rest period and the elevation of the tilt table. BP data were sampled at $1000 \mathrm{~Hz}$ via an A/D converter, and data were stored, together with ECG data, in a computer using Tonam $2 \mathrm{C}$ software. Pulse pressure was calculated as SBP-DBP ( $\mathrm{mmHg})$.

\section{Measurement of BRS}

We used the sequence technique $[22,23]$ to obtain BRS $(\mathrm{ms} / \mathrm{mmHg})$. Briefly, SBP and RR interval were determined beat-to-beat from the digitalized data using the Tonam2C software; sequences of three or more consecutive heart beats, in which SBP consistently increased or decreased and heart beats consistently decreased or increased, were identified, and the slope of the regression line relating the SBP to RR interval was calculated for each of these sequences. Sequences with a correlation coefficient of the relationship between the SBP and RR interval of greater than 0.85 were selected, and for each sequence the slope of the regression line was determined to reflect BRS. BRS value was determined as the average value of all the slopes obtained during $10 \mathrm{~min}$ previous to HUT and during $10 \mathrm{~min}$ of the HUT.

\section{Blood analysis}

Blood samples were centrifuged at $1420 \times \mathrm{g}$ for $10 \mathrm{~min}$ at $4{ }^{\circ} \mathrm{C}$. The separated plasma was frozen at $-35^{\circ} \mathrm{C}$, and was sent to a laboratory (SRL Corporation, Nagoya, Japan) for analysis of plasma concentration of adrenaline, noradrenaline, vasopressin, and renin. Plasma adrenaline and noradrenaline were measured using an HPLC method. Plasma vasopressin was measured using a double-antibody radioimmunoassay. Plasma renin concentration was measured using a solid phase radioimmunoassay method.

\section{Statistical analysis}

Data are expressed as mean \pm standard error of mean (SE). Time-serial data of HR, BP (SBP and DBP), and pulse pressure were analyzed by averaging every $30 \mathrm{~s}$. Timeserial change was tested using one-way repeated measures analysis of variance (ANOVA). Differences between preHUT value and the value at various time points in timeserial data were tested using Dunnett inequalities. Differences between nurse and control groups for HR, SBP, $\mathrm{DBP}$, and pulse pressure were tested using two-way repeated measures ANOVA.
Differences between two groups for BRS during the preHUT and during the HUT and for the changes in BRS by HUT were tested by the Mann-Whitney test. Hormone concentrations at pre-HUT and at the end of HUT were compared by the Wilcoxon test. Changes in hormone concentrations by HUT were compared between the two groups using the Mann-Whitney test. Analysis of confounding factor was performed using ANCOVA.

A significance level of $p<0.05$ was used for all tests. The statistical software used was IBM SPSS Statistics Ver. 19 for Windows.

\section{Results}

All 12 nurse subjects completed the HUT test with no presyncopal signs or symptoms, while two out of 12 control subjects discontinued the test because of hypotension and discomfort. One out of the two subjects who discontinued the test repeated it again on a later day, and completed it safely. Consequently, the data from the 12 nurse subjects (aged $29.5 \pm 1.2$ ) and 11 control subjects (aged $24.1 \pm 1.0$ ) were available for analysis. The age of the subjects was significantly different between the two groups ( $p=0.005$, Table 1 ), but the height, weight, and BMI did not differ significantly (Table 1).

The pre-HUT (baseline) values of HR, SBP, DBP, and pulse pressure were not significantly different between the nurse and control groups (Table 2). Time-serial data of HR, SBP, and DBP were expressed as the change from the pre-HUT (time 0 ), i.e., $\Delta$ value.

The $\Delta \mathrm{HR}$, in both the nurse and control groups, rapidly increased during the first $1 \mathrm{~min}$, and then slowly increased up until $2.5 \mathrm{~min}$ (Fig. 1a). The increase from the pre-HUT value was significant at any point of time after $1 \mathrm{~min}$ in both groups. The change during $10 \mathrm{~min}$ of HUT was statistically significant $(p<0.001$ for the nurse group and

Table 1 Physical and habitual characteristics of subjects

\begin{tabular}{lll}
\hline Variable & Nurse & Control \\
\hline Age (years) & $29.5 \pm 1.2^{*}$ & $24.1 \pm 1.0$ \\
Height $(\mathrm{cm})$ & $158.8 \pm 2.0$ & $158.4 \pm 1.2$ \\
Weight $(\mathrm{kg})$ & $56.1 \pm 2.0$ & $53.7 \pm 3.0$ \\
BMI & $22.2 \pm 0.7$ & $21.3 \pm 1.1$ \\
Experience as a nurse (years) & $7.5 \pm 1.2$ & 0 \\
Smoking habit (years) & 0 & 0 \\
Habit of exercise (years) & 0 & 0 \\
\hline
\end{tabular}

Values are mean $\pm \mathrm{SE}$

$* p<0.01$ compared with control 
Table 2 Changes in hormones and cardiovascular parameters by HUT

\begin{tabular}{|c|c|c|c|c|c|c|}
\hline \multirow[t]{2}{*}{ Variable } & \multicolumn{3}{|l|}{ Nurse } & \multicolumn{3}{|l|}{ Control } \\
\hline & Pre-HUT (a) & End of HUT (b) & Increment $(b-a)$ & Pre-HUT (c) & End of HUT (d) & Increment $(\mathrm{d}-\mathrm{c})$ \\
\hline Adrenaline (pg/ml) & $23.8 \pm 3.1$ & $48.7 \pm 5.8^{*}$ & $24.9 \pm 3.5 \dagger$ & $31.5 \pm 7.1$ & $75.3 \pm 13.4^{*}$ & $43.8 \pm 7.0$ \\
\hline Noradrenaline (pg/ml) & $139.3 \pm 19.2$ & $380.3 \pm 32.8^{*}$ & $241.0 \pm 25.4 \dagger$ & $174.7 \pm 28.2$ & $352.2 \pm 34.2 *$ & $177.5 \pm 18.2$ \\
\hline $\operatorname{Renin}(\mathrm{pg} / \mathrm{ml})$ & $7.5 \pm 0.89$ & $8.1 \pm 1.5^{*}$ & $0.8 \pm 0.8$ & $7.1 \pm 0.5$ & $7.8 \pm 0.6^{*}$ & $0.7 \pm 0.3$ \\
\hline Vasopressin (pg/ml) & $1.8 \pm 0.2$ & $3.9 \pm 0.8^{*}$ & $2.1 \pm 0.6$ & $1.4 \pm 0.1$ & $2.5 \pm 0.3^{*}$ & $1.1 \pm 0.3$ \\
\hline HR (bpm) & $67.8 \pm 2.6$ & $81.9 \pm 3.4$ & & $65.8 \pm 3.7$ & $77.2 \pm 4.2$ & \\
\hline $\mathrm{SBP}(\mathrm{mmHg})$ & $125 \pm 4.7$ & $142.7 \pm 4.7$ & & $131.5 \pm 6.3$ & $140.7 \pm 6.3$ & \\
\hline DBP $(\mathrm{mmHg})$ & $79 \pm 5.8$ & $100.7 \pm 4.9$ & & $88.7 \pm 5.9$ & $104.1 \pm 5.1$ & \\
\hline
\end{tabular}

See the text for the difference between groups in the increment from pre-HUT to end of HUT. Values are mean $\pm \mathrm{SE}$

$* p<0.05$ compared with pre-HUT; $\dagger p<0.05$ compared with control subjects

$p<0.001$ for the control group, one-way ANOVA). However, the difference between the two groups was not statistically significant over the 10-min period of HUT.

The response of $\Delta \mathrm{SBP}$ to HUT was different between the groups (Fig. 1b). As for the control group, the $\Delta \mathrm{SBP}$ did not change significantly during the first 1 min of HUT. Afterward, it rapidly increased until reaching 2 min of HUT, and subsequently leveled off at a level of about $15-20 \mathrm{mmHg}$ above the pre-HUT value. $\Delta$ SBP was maintained at this level for about $4 \mathrm{~min}$, and subsequently declined gradually toward the end of HUT. This serial change was significant ( $p<0.001$, one-way ANOVA). In the nurse group, the $\triangle \mathrm{SBP}$ did not change during the first $30 \mathrm{~s}$, but rapidly increased until reaching $1 \mathrm{~min}$ of HUT, and subsequently leveled off at a level of $20-30 \mathrm{mmHg}$ above the pre-HUT value. This level was maintained until $2.5 \mathrm{~min}$, and finally declined steadily. This serial change in the nurse group was also significant $(p<0.001$, one-way ANOVA). Two-way ANOVA revealed that $\triangle \mathrm{SBP}$ was significantly greater in the nurse group than in the control group for the period of time between 0 and $2 \mathrm{~min}$ ( $p=0.028)$, but was not significantly greater for the period of time between 0 and $10 \mathrm{~min}$.

The $\triangle \mathrm{DBP}$ showed a similar trend as the $\triangle \mathrm{SBP}$, in both the nurse and control groups (Fig. 1c), with significant changes during $10 \mathrm{~min}$ of HUT for each group $(p<0.001$ for the control group and $p=0.005$ for the nurse group, one-way ANOVA). However, no significant difference between the nurse and control groups was observed in the early stage, as well as during the 10-min HUT period.

The pulse pressure was also different between the groups (Fig. 1d). In the control group, the serial change during $10 \mathrm{~min}$ of HUT was significant $(p=0.009$, oneway ANOVA), and the change from the pre-HUT value was significantly lower at 1 and $1.5 \mathrm{~min}(p<0.001$ and $p=0.001$, respectively). In the nurse group, the serial change during 10 min of HUT was not significant, and the difference from the pre-HUT value was not significant at any point of time. Two-way ANOVA revealed that pulse pressure was significantly greater in the nurse group compared with the control group between 1 and $2.5 \mathrm{~min}$ ( $p=0.038)$.

BRS value during pre-HUT was significantly lower in the nurse group as compared with the control group ( $p=0.010$; Fig. 2), and BRS during HUT was also significantly lower in the nurse group $(p=0.003)$. Furthermore, in the nurse group, the BRS was decreased significantly by HUT $(p<0.001)$, whereas in the control group BRS tended to be decreased by HUT $(p=0.062)$. Nevertheless, the decrease of BRS by HUT was not significantly different between the groups, indicating that the magnitude of the response of BRS to HUT was not different between groups.

For each hormone (Table 2), the pre-HUT value was not different between the groups. However, each hormone increased significantly during HUT. Accordingly, we analyzed the increment of each hormone as reflecting the degree of the response to HUT. The results indicated that the increment for noradrenaline was significantly greater $(p=0.045)$ and that for adrenaline was significantly smaller $(p=0.036)$ in the nurse group compared with the control group, while the increment for vasopressin and for renin did not differ significantly between the two groups.

\section{Discussion}

The main results of this study are as follows: (1) $\triangle \mathrm{SBP}$ showed an overshoot response during HUT in both the nurse and control groups; (2) the overshoot response of $\Delta \mathrm{SBP}$ was significantly greater in the nurse group than in 

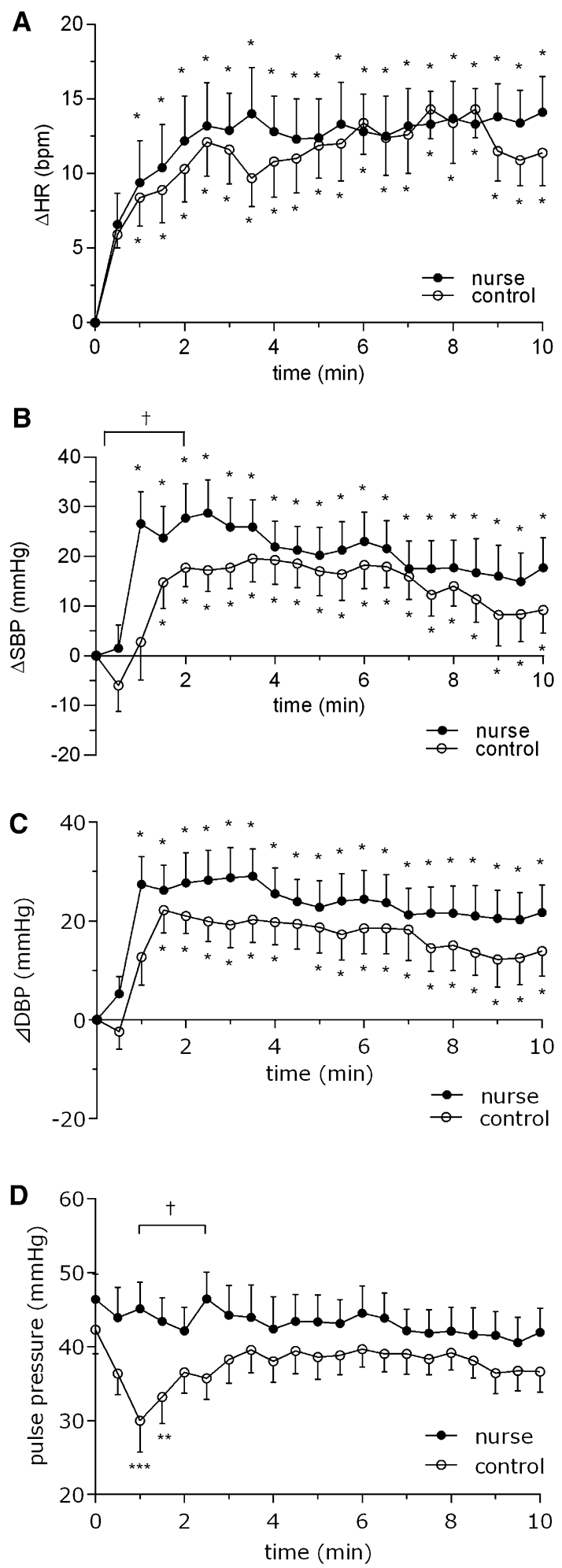

Fig. 1 HR (a), SBP (b), DBP (c), and pulse pressure (d) responses to HUT. HR, SBP, and DBP are represented as the change from the preHUT value $(\Delta$ value). $* p<0.05, * * p<0.01$, and $* * * p<0.001$ compared with pre-HUT (time 0 ); $\dagger p<0.05$ compared with control group, see text for the details. $\mathrm{bpm}$ beats/min. Values are mean $\pm \mathrm{SE}$

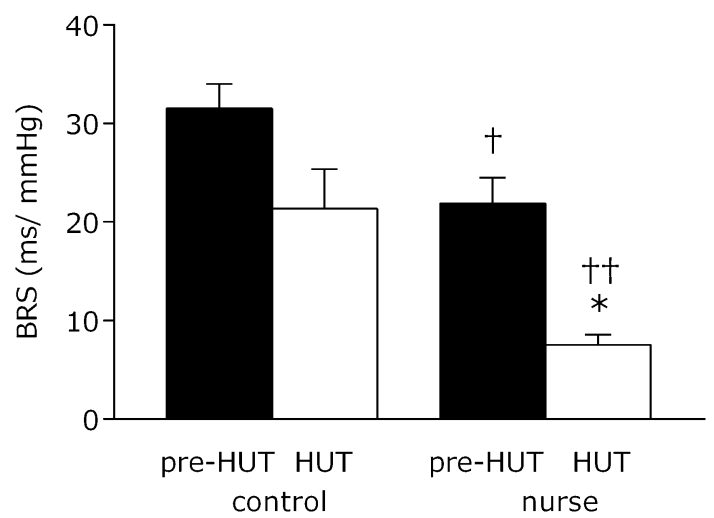

Fig. 2 Changes in baroreflex sensitivity (BRS) by HUT. * $p<0.01$ compared with pre-HUT; $\dagger p<0.05, \dagger \dagger p<0.01$ compared with control. Values are mean $\pm \mathrm{SE}$

the control group during the first 2 min of HUT; (3) a reduction in pulse pressure occurred during 1-2.5 min of HUT in the control group, but it was absent in the nurse group; (4) BRS was decreased by HUT in the nurse group, while it tended to be decreased in the control group; and (5) the increment of plasma noradrenaline concentration during HUT was significantly greater in the nurse group than the control group.

\section{Responses to HUT}

Generally, SBP hardly changes during HUT, although it may decrease transiently at an early stage owing to the shift of blood to the lower part of the body caused by orthostatic stress. This reduction is minimal or even absent in a passive HUT, because of various compensatory mechanisms including the arterial baroreflex $[4,5]$.

Surprisingly, we observed both in the nurse and the control groups that $\triangle \mathrm{SBP}$ produced an overshoot in BP, which endured from the initial 1 or $1.5 \mathrm{~min}$ until the end of HUT. Furthermore, $\triangle \mathrm{SBP}$ was significantly higher during the first 2 min of HUT in the nurse group than in the control group. Orthostatic tolerance depends on physical factors such as height, plasma volume, arterial and cardiopulmonary baroreflex sensitivity, cardiac filling, or stroke volume [18, 24-26]. Furthermore, brain blood flow is a critical factor for syncope [27, 28]. A higher BP during HUT should be advantageous for maintaining the arterial $\mathrm{BP}$ in the brain above the lowest limit of the $\mathrm{BP}$ range for autoregulation $(60-140 \mathrm{mmHg}$ for mean BP [27]). Thus, our observations suggest that the nurses had higher orthostatic tolerance than the control subjects, at least in the early stage of HUT. We did not directly measure orthostatic tolerance, but the high tolerance in nurses is 
confirmed by our observation that all 12 nurse subjects were able to complete the HUT test, while two out of 12 control subjects failed to complete it.

Overshooting responses of SBP during HUT somewhat differed from normal responses reported so far. However, similar BP responses during HUT were reported in healthy subjects by Gabbett et al. [29], who conducted a $90^{\circ}$ HUT test on young males, and observed that a BP overshoot in an early stage of HUT to about $10 \mathrm{mmHg}$ above the supine value. It has been widely accepted that considerable individual variations exist in orthostatic tolerance [25, 26, 30, 31]. Accordingly, we assume that the BP overshoot response observed in both subject groups probably reflects great individual variations in orthostatic tolerance.

Noradrenaline usually increases in response to HUT [32, 33 ], as is noted in this study. Importantly, the increment in noradrenaline concentrations by HUT was significantly greater in the nurse group than the control group. Noradrenaline in the blood stream is derived from a spillover from postganglionic sympathetic nerve fiber endings [34], and blood noradrenaline concentration is linearly related to the degree of vasoconstrictor nerve activity [35]. Our observations indicate that the greater BP overshoot response observed in the nurse group was closely related to greater sympathetic (noradrenergic) vasoconstrictor nerve activity.

\section{Hemodynamic mechanism}

Hemodynamics is an important element for the maintenance of BP during orthostatic challenge [4]. Since HR increase reached a significant level at $1 \mathrm{~min}$ of HUT, autonomic compensation would have developed at this time point. At $0.5 \mathrm{~min}$ of HUT, SBP, DBP, and pulse pressure did not differ significantly from pre-HUT in both groups, suggesting that the hemodynamic mechanism in this initial stage of HUT should not differ between groups.

In the early stage (1-2 min), in which a compensatory mechanism occurred, SBP and DBP were elevated in both groups. Coincidentally, vasoconstriction should have occurred in this stage. Interestingly, while control subjects showed a transient decrease in pulse pressure at 1 and 1.5 min of HUT, nurse subjects showed no such responses. Given that pulse pressure is a function of stroke volume [36], this observation suggests that the reduction in stroke volume produced by HUT was smaller in the nurse group compared with the control group. Thus, it is possible that stroke volume was an important factor in the greater response of SBP observed in nurse subjects during the early stage of HUT.

In the prolonged stage of 2-10 min, SBP and DBP maintained higher values than the pre-HUT, but with no apparent difference between the groups. Pulse pressure also did not differ between the groups. Vasoconstriction probably maintained a higher level, as is evidenced by the higher noradrenaline value at the end of HUT.

\section{Compensatory mechanism}

\section{Arterial baroreflex}

The prolonged elevation of BP observed in both subject groups, which endured until the end of HUT, was accompanied by vasoconstriction. The mechanism of the BP elevation is difficult to explain based on a single, fixed baroreceptor function curve because, if BP fluctuation occurred, it should be buffered rapidly by arterial baroreflex [37], and a BP elevation would not be sustained. However, such a prolonged elevation of BP during HUT can be totally explained by the "resetting" of the arterial baroreflex [14-18, 38, 39], which predicts the rightward and upward shift of the arterial baroreflex curve in this study.

Some recent studies have demonstrated that baroreflex resetting occurs upon standing, supporting our explanation. Schwartz et al. [18] demonstrated in humans that the baroreflex function curve shifts rightward and upward during HUT to increase vasoconstriction, although they did not report BP elevation. Kamiya et al. [17] proved in an animal model that HUT resets the baroreflex control to increase sympathetic activity to a higher level.

Thus, the theory of resetting is attractive to describe the whole mechanism of the prolonged elevation of BP, but it is still defective in direct evidence. For full elucidation of the baroreflex function curve, invasive measurements such as the Oxford method or the neck chamber method $[16,18$, 37] should be undertaken.

\section{Other compensatory mechanisms}

The prolonged BP elevation is otherwise explained by a compensatory mechanism other than the arterial baroreflex, such as the cardiopulmonary baroreflex $[6,7]$ or vestibulosympathetic reflex [8]. It is generally accepted that, in a prolonged stage ( $>2 \mathrm{~min}$ ) of orthostasis, BP maintenance is supported by humoral factors [4, 5]. However, a recent study has demonstrated that vasoconstrictor nerve activity is still maintained when an upright posture is prolonged (e.g., for $45 \mathrm{~min}$ ) [40]. This result clearly indicates an important role of a compensatory mechanism in the BP elevation during the prolonged stage of HUT in this study. This result supports the view that the BP elevation during the prolonged stage of HUT was mediated by the arterial baroreflex ("resetting"), but it also proves the possibility that the BP elevation was mediated by the compensatory mechanism other than the arterial baroreflex.

Additionally, it is probable that the compensatory mechanism other than the arterial baroreflex contributed to the transient BP elevation that occurred during the first 2 min 
of HUT, which produced a significant difference between the nurse and control subjects. The rationale is the general view that the circulatory response during the early stage $(<2 \mathrm{~min})$ of upright posture is governed by the neurovascular system $[4,5]$. If so, however, with the present data it is impossible to detect which mechanism was involved.

\section{BRS response}

BRS was decreased by HUT in the nurse group, while it tended to be decreased in the control group. The majority of studies have shown that BRS is reduced during HUT [4143], while a few studies have reported that it is augmented [30]. Thus, BRS changes in this study were virtually consistent with the former results. However, the physiological implications of the lower BRS during HUT are unclear. One plausible explanation is that the reduced BRS during HUT resulted from an interaction of the arterial baroreflex with the cardiopulmonary baroreflex [6]. Furthermore, the reduction in BRS appears to contribute at least in part to the BP elevation during HUT, because it diminishes the buffering effect of the baroreflex on BP, and thereby allowing the BP to greatly fluctuate [44]. This mechanism may be more effective in nurses, because nurses have a lower BRS during HUT. However, it is still questionable whether the reduction in BRS is essential for maintaining a higher BP during HUT.

\section{Confounding factors}

In this study, BRS was lower in the nurse group than in the control group. Given that BRS decreases as age increases [43], and thus is a confounding factor, we examined the correlation of age with BRS using regression analysis. We found no significant correlation in either group between age and BRS. The same results were obtained for correlations between age and SBP, DBP, pulse pressure, and noradrenaline concentration. Consequently, adjustment by age was deemed unnecessary. We also examined the relationship between BMI and each of the variables, and obtained the same results.

\section{Limitations and perspectives}

This study had several limitations. First, we did not evaluate the physical fitness of the subjects, which can affect orthostatic tolerance [45]; thus, we did not measure $V_{\mathrm{o}_{2 \max }}$. Second, we did not measure blood volume, which also affects orthostatic tolerance [11, 12]. Given that physical training may increase blood volume, moderately fit persons should attain a higher tolerance to orthostasis because of a large blood volume [11].

Third, we used the sequence method for evaluating BRS, which is safety for human subjects and appropriate to use in field studies because it measures noninvasively under resting conditions. The sequence method estimates the response of the cardiac component of the arterial baroreflex, and it is questionable whether it exactly reflects the vascular component [30, 42]. The vascular component of the arterial baroreflex can be estimated accurately by measuring other variables such as vascular resistance [30] or muscle sympathetic nerve activity [18].

This study is a pilot study examining the orthostatic tolerance of nurses, and thus gave no insight into the direct physiological or physical factors responsible for the cardiovascular responses to orthostatic stress. Furthermore, the process by which cardiovascular responses developed in nurses (whether they occurred inherently or as a consequence of the long-term adaption to nursing works) was not identified. Elucidation of this process would contribute to understanding the mechanisms of individual variations in orthostatic tolerance existing in healthy persons.

Our findings have implications for the occupational health of nurses and other workers who undertake similar work activities. Future studies in this area should focus on designing methods for improving orthostatic tolerance in such workers who have inappropriately poor orthostatic tolerance. Once established, these methods would greatly contribute to preventing such workers from dropping out of their chosen career.

In conclusion, although nurse subjects had a lower BRS than control subjects, they were able to effectively maintain BP during HUT, suggesting that nurse subjects had higher orthostatic tolerance. The better maintenance of BP in nurse subjects appeared to be associated with a compensatory mechanism other than the arterial baroreflex and/ or a hemodynamic mechanism.

Acknowledgments The authors would like to express their thanks to Prof. Humio Kobayashi, Department of Health and Psychosocial Medicine, Aichi Medical University, and Prof. Satoshi Iwase, Department of Physiology, Aichi Medical University, for helpful advice and for providing the facilities for the experiments. This study was conducted under the auspices of nurses of the Department of Nursing, Japanese Red Cross Nagoya First Hospital. It was supported in part by Academic Research Grants from the Daiko Foundation (2010) and Policy-Based Medical Service Foundation (2010).

Conflict of interest The authors declare that they have no conflict of interest.

\section{References}

1. Lee YS, Huang YC, Kao YH. Physical activities and correlates of clinical nurses in Taipei municipal hospitals. J Nurs Res. 2005;13:281-92.

2. Abbey MA, Chaboyer W, Mitchell M. Understanding the work of intensive care nurses: a time and motion study. Aust Crit Care. 2012;25:13-22. 
3. Douglas S, Cartmill R, Brown R, Hoonakker P, Slage J, van Roy $\mathrm{KS}$, et al. The work of adult and pediatric intensive care unit nurses. Nurs Res. 2013;62:50-8.

4. Wieling W, van Lieshout JJ. Maintenance of postural normotension in humans. In: Low PA, Benarroch EE, editors. Clinical autonomic disorders. 3rd ed. Philadelphia: Lippincott Williams \& Wilkins; 2008. p. 57-67.

5. Rowell LB. Human Cardiovascular Control. New York: Oxford University Press; 1993.

6. Victor RG, Mark AL. Interaction of cardiopulmonary and carotid baroreflex control of vascular resistance in humans. J Clin Invest. 1985;76:1592-8.

7. Pawelczyk JA, Raven PB. Reductions in central venous pressure improve carotid baroreflex responses in conscious men. Am J Physiol. 1989;257(5 P2):H1389-95.

8. Carter JR, Ray CA. Sympathetic responses to vestibular activation in humans. Am J Physiol Regul Integr Comp Physiol. 2008;294:R681-8.

9. Henriksen O, Sejrsen P. Local reflex in microcirculation in human skeletal muscle. Acta Physiol Scand. 1977;99:19-26.

10. McCarthy JP, Bamman MM, Yelle JM, LeBlanc AD, Rowe RM, Greenisen MC, et al. Resistance exercise training and the orthostatic response. Eur J Appl Physiol. 1997;76:32-40.

11. Mtinangi BL, Hainsworth R. Effects of moderate exercise training on plasma volume, baroreceptor sensitivity and orthostatic tolerance in healthy subjects. Exp Physiol. 1999;84:121-30.

12. El-Sayed H, Hainsworth R. Relationship between plasma volume, carotid baroreceptor sensitivity and orthostatic tolerance. Clin Sci. 1995;88:463-70.

13. Tatro DL, Dudley GA, Convertino VA. Carotid-cardiac baroreflex response and LBNP tolerance following resistance training. Med Sci Sports Exerc. 1992;24:789-96.

14. Sagawa K. Baroreflex control of systemic arterial pressure and vascular bed. In: Shepherd JT, Abboud FM, editors. Handbook of physiology, sec. 2, the cardiovascular system, vol. III. Bethesda: American Physiological Society; 1983. p. 453-96.

15. DiCarlo SE, Bishop VS. Central baroreflex resetting as a means of increasing and decreasing sympathetic outflow and arterial pressure. Ann N Y Acad Sci. 2001;940:324-37.

16. Raven PB, Fadel PJ, Ogoh S. Arterial baroreflex resetting during exercise: a current perspective. Exp Physiol. 2006;2006(91):37-49.

17. Kamiya A, Kawada T, Yamamoto K, Michikami D, Ariumi H, Uemura K, et al. Resetting of the arterial baroreflex increases orthostatic sympathetic activation and prevents postural hypotension in rabbits. J Physiol. 2005;566(1):237-46.

18. Schwartz CE, Stewart JM. The arterial baroreflex resets with orthostasis. Front Physiol. 2012;3:1-10.

19. Benditt DG, Ferguson DW, Grubb BP, Kapoor WN, Kugler J, Lerman BB, et al. Tilt table testing for assessing syncope. J Am Coll Cardiol. 1996;28:263-75.

20. Parati G, Casadei R, Groppelli A, Di Rienzo MD, Mancia G. Comparison of finger and intra-arterial blood pressure monitoring at rest and during laboratory testing. Hypertension. 1989;13(6 Pt 1):647-55.

21. Wieling W. Non-invasive continuous recording of heart rate and blood pressure in the evaluation of neurocardiovascular control. In: Bannister R, Mathias CJ, editors. Autonomic failure. Oxford: Oxford University Press; 1992. p. 291-311.

22. Di Rienzo M, Castiglioni P, Iellamo F, Volterrani M, Pagani M, Mancia G, et al. Dynamic adaptation of cardiac baroreflex sensitivity to prolonged exposure to microgravity: data from a 16 day spaceflight. J Appl Physiol. 2008;105:1569-75.

23. La Rovere MT, Pinna GD, Raczak G. Baroreflex sensitivity: measurement and clinical implications. Ann Noninvasive Electrocardiol. 2008;13:191-207.
24. Ludwig DA, Convertino VA. Predicting orthostatic intolerance: physiology or physics? Aviat Space Environ Med. 1994;65:404-11.

25. Convertino VA, Sather TM. Vasoactive neuroendocrine responses associated with tolerance to lower body negative pressure in humans. Clin Physiol. 2000;20:177-84.

26. Fu Q, Witkowski S, Levine BD. Vasoconstrictor reserve and sympathetic neural control of orthostasis. Circulation. 2004;110:2931-7.

27. Franco Folino A. Cerebral autoregulation and syncope. Prog Cardiovasc Dis. 2007;50:49-80.

28. Harms MPM, Colier WNJM, Wieling W, Lenders JWM, Secher $\mathrm{NH}$, van Lieshout JJ. Orthostatic tolerance, cerebral oxygenation, and blood velocity in humans with sympathetic failure. Stroke. 2000;31:1608-14.

29. Gabbett TJ, Weston SB, Barrett RS, Gass GC. Cardiovascular regulation during head-up tilt in healthy 20 to 30 -year-old and 70 to 75-year-old men. Clin Sci (Lond). 2001;100:199-206.

30. Cooper VL, Hainsworth R. Effects of head-up tilting on baroreceptor control in subjects with different tolerances to orthostatic stress. Clin Sci. 2002;103:221-6.

31. Cote AT, Bredin SS, Phillips AA, Warburton DE. Predictors of orthostatic intolerance in healthy young women. Clin Invest Med. 2012;35:E65-74.

32. Ziegler MG, Lake R, Kopin IJ. The sympathetic-nervous-system defect in primary orthostatic hypotension. $\mathrm{N}$ Eng $\mathrm{J}$ Med. 1977;296:293-7.

33. László Z, Rössler A, Hinghofer-Szalkay HG. Cardiovascular and hormonal changes with different angles of head-up tilt in men. Physiol Res. 2001;50:71-82.

34. Esler M, Jackman G, Bobik A, Leonard P, Kelleher D, Skews H, et al. Norepinephrine kinetics in essential hypertension. Defective neuronal uptake of norepinephrine in some patients. Hypertension. 1981;3:149-56.

35. Wallin BG, Mörlin C, Hjemdahl P. Sympathetic activity and plasma noradrenaline concentrations during static exercise in normotensive and hypertensive subjects. Acta Physiol Scand. 1987;129:489-97.

36. Convertino VA, Cook WH, Holcomb JB. Arterial pulse pressure and its association with reduced stroke volume during progressive central hypovolemia. J Trauma. 2006;61:629-34.

37. Schwartz CE, Medow MS, Messer Z, Stewart JM. Spontaneous fluctuation indices of the cardiovagal baroreflex accurately measure the baroreflex sensitivity at the operating point during upright tilt. Am J Physiol Regul Integr Comp Physiol. 2013;304:R1107-13.

38. Rowell LB, O'Leary DS. Reflex control of the circulation during exercise: chemoreflexes and mechanoreflexes. J Appl Physiol. 1990;69:407-18.

39. Sato T. New analytic framework for understanding sympathetic baroreflex control of arterial pressure. Am J Physiol. 1999;276:H2251-61.

40. Fu Q, Shook RP, Okazaki K, Hasting JL, Shibata S, Conner CL, et al. Vasomotor sympathetic neural control is maintained during sustained upright posture in humans. J Physiol. 2006;577(2):679-87.

41. Kardos A, Rudas L, Simon J, Gingl Z, Csanády M. Effect of postural changes on arterial baroreflex sensitivity assessed by the spontaneous sequence method and valsalva manoeuvre in healthy subjects. Clin Auton Res. 1997;7:143-8.

42. O'Leary DD, Kimmerly DS, Cechetto AD, Shoemaker JK. Differential effect of head-up tilt on cardiovagal and sympathetic baroreflex sensitivity in humans. Exp Physiol. 2003;88(6): 769-74.

43. Laitinen T, Hartikainen J, Vanninen E, Niskanen L, Geelen G, Länsimies E. Age and gender dependency of baroreflex sensitivity in healthy subjects. J Appl Physiol. 1998;84:576-83. 
44. Fauvel JP, Cerutti C, Quelin P, Laville M, Gustin MP, Paultre $\mathrm{CZ}$, et al. Mental stress-induced increase in blood pressure is not related to baroreflex sensitivity in middle-aged healthy men. Hypertension. 2000;35:887-91.
45. Levine BD, Buckey JC, Fritsch JM, Yancy CW Jr, Watenpaugh DE, Snell PG, et al. Physical fitness and cardiovascular regulation: mechanisms of orthostatic intolerance. J Appl Physiol. 1991;70:112-22. 